\title{
Active Biomechanics of Sensory Hair Bundles
}

\author{
Dolores Bozovic ${ }^{1,2}$ \\ ${ }^{1}$ Department of Physics and Astronomy, University of California, Los Angeles, California 90095-1547 \\ ${ }^{2}$ California NanoSystems Institute, University of California, Los Angeles, California 90095-1547 \\ Correspondence: bozovic@physics.ucla.edu
}

During the detection of sound, hair bundles perform a crucial step by responding to mechanical deflections and converting them into changes in electrical potential that subsequently lead to the release of neurotransmitter. The sensory hair bundle response is characterized by an essential nonlinearity and an energy-consuming amplification of the incoming sound. The active response has been shown to enhance the hair bundle's sensitivity and frequency selectivity of detection. The biological phenomena shown by the bundle have been extensively studied in vitro, allowing comparisons to behaviors observed in vivo. The experimental observations have been well explained by numerical simulations, which describe the cellular mechanisms operant within the bundle, as well as by more sparse theoretical models, based on dynamical systems theory.

$\mathrm{T}_{\mathrm{ici}}^{\mathrm{h} \text { ed }}$ he auditory system presents us with a biologcal detector having impressive sensitivity, dynamic range, and frequency selectivity (Hudspeth 2014). Near detection thresholds, the inner ear responds to displacements of the basilar membrane that are on the order of Angstroms ( $)$. This detection is performed in the presence of thermal noise, estimated to lead to fluctuations of an order of magnitude higher. The property appears to be universal, as comparable detection thresholds have been found across a wide variety of species (Manley 2000). The mechanisms underlying this sensitivity continue to fascinate the research community in fields ranging from biology to physics (Reichenbach and Hudspeth 2014).

Hair cells are the biological sensors that detect mechanical vibrations that reach the inner ear (LeMasurier and Gillespie 2005; Vollrath et al. 2007). On the apical surface of a hair cell, 20-300 actin-filled microvilli-like structures called sterocilia protrude from the cell body to form the hair bundle. This organelle contains mechanically sensitive ion channels, enabling it to transduce deflections of the stereocilia into electrical signals. Preparations of living hair cells have been obtained from different end organs and from various species, allowing in vitro studies of their response. Modern optical techniques allow the detection of nanometer and smaller deflections of hair bundles, and probes have been developed for applying and measuring forces in the piconewton range. The response of a single hair cell can moreover be compared with macroscopic observations of in vivo phenomena.

Editors: Guy P. Richardson and Christine Petit

Additional Perspectives on Function and Dysfunction of the Cochlea available at www.perspectivesinmedicine.org

Copyright (C 2019 Cold Spring Harbor Laboratory Press; all rights reserved; doi: 10.1101/cshperspect.a035014

Cite this article as Cold Spring Harb Perspect Med 2019;9:a035014 
D. Bozovic

\section{NONLINEAR MECHANICS}

Nonlinearity in the response has been extensively shown by in vivo experiments performed on the basilar membrane, and shown to be one of the hallmarks of the acuity of hearing (Robles and Ruggero 2001). Intact and functional hair cells were furthermore shown to be essential components in the nonlinear response.

The mechanical response of an individual hair bundle was likewise shown to be highly nonlinear (Russell et al. 1992). Experiments performed in vitro on preparations of the bullfrog sacculus showed that a hair bundle exposed to direct mechanical stimulation is sensitive to signals as small as $1 \mathrm{~nm}$ (Martin and Hudspeth 2001). Response over an intermediate range of stimulus levels showed one-third power-law growth (Fig. 1), consistent with in vivo observations, while at very weak and very strong drives, the response was linear. In contrast, similar measurements performed at stimulus frequencies far from the characteristic frequency of the cell elicited a linear response over the full range of phys-

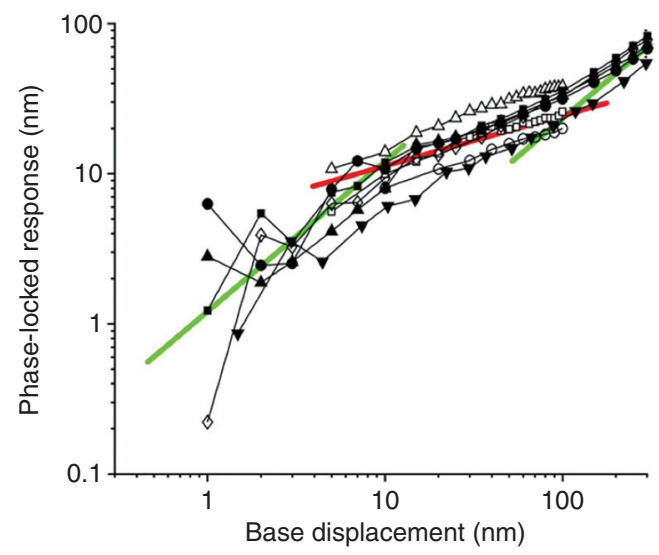

Figure 1. Nonlinear mechanical response of the hair bundle, recorded from a preparation of the bullfrog sacculus. The phase-locked response of the hair bundle was plotted for varying amplitudes of the applied stimulus. The intermediate regime showed a onethird power law (red line), while weak and strong stimuli elicited a linear response (green lines). The plot shows data obtained from different hair bundles. (From Martin and Hudspeth 2001; reprinted, with permission, from the National Academy of Sciences (C) 2001.) iological stimulus intensities. These experiments showed that the power-law nonlinearity observed in the basilar membrane motion is also present at the level of single-cell dynamics.

The phenomenon of distortion product generation also has a counterpart in the mechanical response of a single hair cell (Jaramillo et al. 1993). When hair bundles were mechanically stimulated with waveforms that contained a superposition of two frequencies, the power spectra showed clear peaks at various frequencies corresponding to distortion products. The $\left(2 f_{1}-f_{2}\right)$ difference peak was observed with stimulus amplitudes as small as $12 \mathrm{~nm}$, and was dependent on the presence of an intact transduction process.

In vivo studies have shown that the nonlinearity of the cochlear response disappears following anoxia or postmortem (Dallos 1992), indicating that it is closely linked to an internal active process that requires the consumption of energy. A study performed on hair bundles in vitro elegantly showed that the observed distortion products, also called phantom tones, are signatures of an active process (Barral and Martin 2012). For a passive nonlinearity, the magnitude of the distortion product relative to that of the stimulus tone would grow as the square of the magnitude of the applied force. The measurements instead indicated that the relative level of the distortion was roughly constant, at $\sim 10 \%-20 \%$, over a broad range of applied signals, which is consistent with the behavior of an active oscillator. The level of distortion products was maximal at small separations between the primary tones and decreased with increasing separation.

Although the in vivo response likely involves additional complexities, including interactions along the full auditory organ, the experiments performed in vitro showed that nonlinear effects occur already at a single-cell level. Furthermore, the nonlinear response of the hair bundle, elicited by weak or moderate force, is active in nature.

\section{ACTIVE MOTION}

Thomas Gold (Gold 1948) first introduced the notion that the detection process by the inner 
ear cannot be purely passive. He pointed out that the cochlea operates in a highly viscous environment, with the consequence that any passive motion of the cochlear partition would be overdamped. He posited that an internal active mechanism must pump energy into the system and serve to amplify its motion on a cycle-bycycle basis. Although alternate models have been proposed (van der Heijden 2014), many in vivo studies have supposed the presence of an active amplifier in the cochlea (Dallos 1992), as well as other end organs (Manley and Koppl 1998).

Active mechanics have also been shown in the response of individual hair cells. Hair bundles of a number of species have been shown to display spontaneous oscillations (Crawford and Fettiplace 1985; Martin and Hudspeth 1999). The amplitude of these oscillations typically lies in the range of $\sim 20-100 \mathrm{~nm}$, significantly larger than any thermal fluctuations inherent in the system. This active motility is moreover associated with the opening and closing of the mechanoelectrical transduction channels (Fig. 2 ), because it is abolished by their blockage, as discussed further in this review (Martin et al. 2003; Meenderink et al. 2015).

Several in vitro experiments provided direct proof that the hair bundle oscillations require an active expenditure of energy. These noisy innate oscillations can be readily phase-locked by very weak stimuli (Martin and Hudspeth 1999). A technique using an elastic microfiber of known stiffness controlled by a piezoactuator was developed, allowing one to impose calibrated displacements on the bundle, while simultaneously inferring the forces exerted by the stimulating probe. Integrating the active work performed by the bundle on the fiber yielded an estimate of $\sim 80$ zJ of energy per stimulus cycle. The bundle was shown to deliver power during both the positive and negative phases of the movement.

As a further physical test of whether spontaneous oscillations in vitro are driven by an active process, the response of a bundle was compared with the predictions based on the fluctuation dissipation theorem (Martin et al. 2001; Dinis et al. 2012). This well-known theorem of statistical mechanics states that there is a direct relationship between the response sensitivity of a system and the rate at which fluctuations decorrelate its motion. The theorem applies to all passive systems, linear and nonlinear alike, and its violation provides a strong indication that an active process must be at work. To test whether bundle motion obeys this theorem, the autocorrelation function was computed for the spontaneous oscillations of the bundle. The linear response function was extracted from the measured response evoked by an applied force. According to the fluctuation dissipation theorem, the linear response function of a passive system is related to the power spectrum of its correlation function. With the bundle stimulated by $15 \mathrm{~nm}$ and less, the relation was violated

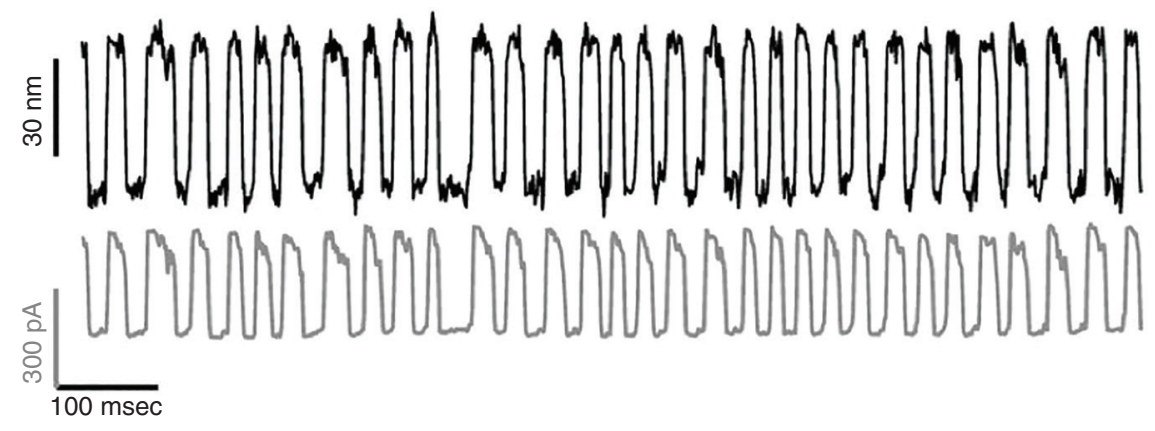

Figure 2. Active bundle motion. Spontaneous oscillations, which occur in the absence of any stimulus, were observed in vitro. Mechanical movements of the bundle (black trace) were closely reflected in the transduction current entering the cell (gray trace). Inward current is plotted in the positive direction, to aid in observing the correlation between the two traces. (From Meenderink et al. 2015; reprinted, with permission, from the Society for Neuroscience (c) 2015.) 
D. Bozovic

at all frequencies. Hence, the oscillation of the bundle was unequivocally proven to be active.

\section{GATING OF THE TRANSDUCTION COMPLEX}

Hair bundles contain the subcellular machinery that performs mechanoelectrical transduction (Hudspeth 2000; Vollrath et al. 2007). The mechanics of hair bundle motion reflect, among other contributions, the stiffness of the stereociliary pivots, located at the basal region of the stereocilia in which their actin core tapers just above the cuticular plate. Within the tapering region, the actin core of the stereocilium is fasciculated around a rootlet, formed of a few tens of more densely packed actin filaments that extend over comparable distances above and below the cuticular plate. The rootlets contribute a significant fraction of the stiffness of the stereociliary pivots (Kitajiri et al. 2010). The cuticular plate itself, a thick (up to $1 \mu \mathrm{m}$ ) meshwork of actin filaments that forms the mechanical support of the hair bundle, also contains structures surrounding the rootlets that likely contribute to this stiffness (Furness et al. 2008). Furthermore, the stereocilia are connected to each other by various filamentous links (see Petit 2018), present at multiple heights, which also contribute to the overall mechanical stiffness of the hair bundle. The arrangement of stereocilia shows a staircase structure reminiscent of an organ pipe, with one axis of symmetry along which the stereocilia increase in height. Along this axis, which also corresponds to the direction of mechanical sensitivity of the bundle, tip links couple the tips of each stereocilium to the shaft of the adjacent taller stereocilium. The tip links in turn are coupled to mechanically gated ion channels, which allow the influx of transduction current into the bundle.

An incoming sound evokes an oscillating motion that deflects the stereocilia in a coherent fashion (Kozlov et al. 2007), and the induced shearing increases the tension in the interconnecting tip links. A compliant element called the gating spring, which may comprise an element in series with the tip link, or the tip link itself, acts on the mechanically sensitive trans- ducer channel to modulate its opening probability (Gillespie et al. 2005). When the channels open, the influx of ions depolarizes the cell. This triggers the opening of voltage-gated calcium channels at synaptic active zones located in the hair cell's basal region, ultimately leading to the release of neurotransmitter to the synaptic boutons of afferent auditory fibers.

A series of in vitro experiments have shown that the gating of the transduction channels is a key component of the nonlinear response of the bundle (Howard and Hudspeth 1988; van Netten et al. 2003). Furthermore, under appropriate concentrations of external cations, the mechanical gating of the channels leads to a region of negative hair bundle stiffness (Martin et al. 2000; Le Goff et al. 2005). Experiments probed the effects of channel gating by measuring the forces required to impose specific displacements on a bundle. These force-displacement curves contained regions of negative stiffness for deflection amplitudes below $\sim 20 \mathrm{~nm}$ (Fig. 3) and of positive stiffness for higher-amplitude deflections. Blockage of the transduction channels eliminated the negative stiffness region and fully linearized the response.

The occurrence of negative stiffness is explained by the gating spring model, which proposes that the opening of the transduction channels is accompanied by a conformational change of the compliant element mentioned above, leading to a 3-7 $\mathrm{nm}$ reduction in the extension of this element between the stereocilia (Markin and Hudspeth 1995). The channel opening is assumed to be stochastic, with a small number of channel states (typically one closed and one open), and the probability of opening to be governed by the Boltzmann distribution (Hudspeth et al. 2000). These assumptions lead to a simple model of the bundle stiffness, which reproduces well the force-displacement relations obtained experimentally, including the region of negative stiffness (Martin et al. 2000).

The location and molecular identity of the gating spring remain unclear and are the topics of ongoing research (Gillespie and Muller 2009; Hudspeth 2014). The stiffness of individual gating springs was estimated according to the gating spring model to be $\sim 0.5 \mathrm{mN} / \mathrm{m}$. However, 
Hair Bundles

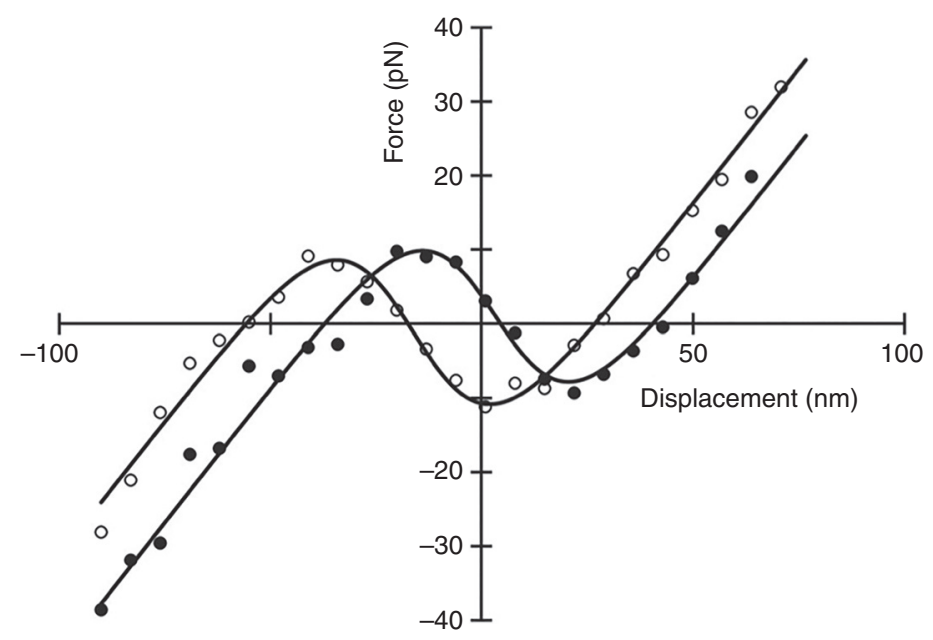

Figure 3. Gating of the transduction channels. The force-displacement curve, obtained from an oscillatory hair bundle of the bullfrog sacculus, showed a region of effective negative stiffness. The response was measured immediately after transitions in the positive (open circle) or negative (full circle) direction and showed a $20 \mathrm{~nm}$ offset. (From Le Goff et al. 2005; reprinted, with permission, from the authors and courtesy of National Academy of Sciences (C) 2005.)

molecular dynamics simulations have indicated that tip links are far too stiff $(\sim 50 \mathrm{mN} / \mathrm{m})$ to constitute the gating spring (Sotomayor et al. 2010). The compliance resides in an element yet to be identified, which lies in series with the tip link and could include the membrane of the stereocilium itself.

\section{THE HAIR CELL'S ADAPTATION PROCESSES}

\section{Molecular Motors and Adaptation}

An adaptation process that reduces the transduction current on sustained deflection of the hair bundle has been shown to exist and was characterized in a number of electrophysiological experiments (Eatock 2000; Fettiplace and Ricci 2003; Gillespie and Cyr 2004). In vestibular hair cells from the mouse, the actin-binding, ATP-dependent molecular motor myosin $1 \mathrm{c}$ was shown to be implicated in the phenomenon (Holt et al. 2002); other motors, such as myosin VIIa, have been proposed to be involved in the adaptation process in cochlear hair cells, although the identity of an adaptation motor in the cochlea is still debated. Besides affecting the ionic influx, adaptation was also shown to exert a clear effect on the mechanical response of the hair bundle (Hudspeth and Gillespie 1994; Gillespie and Corey 1997; Fettiplace and Hackney 2006). The force-displacement curve was observed to shift during prolonged offsets (Martin et al. 2000), consistent with the presence of molecular motors in series with the channels (Fig. 3).

The adaptation mechanism is assumed to involve the coupling of the molecular motors to the tip links and to the stereocilium actin core, formed of a polarized array of parallel actin filaments. The polarity of the actin is such that the ATP-powered motion of the myosin motors proceeds toward the tip of the stereocilium, maintaining the tip-link tension under resting conditions. During a positive deflection of the hair bundle (toward the taller stereocilia), if sufficient mechanical force is exerted by the tip link, the transduction channels open and $\mathrm{Ca}^{2+}$ enters the cell. This leads to a decrease of the calcium-dependent actin-binding affinity of the myosin motors, which allows the array of adaptation motors to slide downward. Thus, a positive bundle deflection causes the slippage of molecular motors and the release of tip-link tension, leading to a decrease of the transduction current and to a positive shift in the resting 


\section{Bozovic}

position of the bundle. Likewise, negative deflection leads to the closing of the transduction channels and a decrease of $\mathrm{Ca}^{2+}$ influx into the stereocilia, thus allowing the myosin array to climb and induce a negative offset in the bundle position. In this model, physical movement of the upper ends of the tip links along the stereocilia, mediated by the myosin adaptation motors, is proposed to fine-tune the tip-link tension and to continuously position the bundle in the regime of its optimal sensitivity.

\section{Calcium Feedback}

An additional phenomenon, known as fast adaptation, or rapid reclosure of the transducer channels, was also observed in the evoked response of a hair bundle (Holt and Corey 2000). This form of adaptation corresponds to a rapid ( $\sim 1 \mathrm{msec}$ or shorter) reduction in the current influx into the cell, and manifests itself mechanically in the form of an equally rapid twitch of the bundle position (Benser et al. 1996). The twitches were shown to be evoked either by displacements with mechanical probes or in response to membrane potential changes. Fast adaptation is well-characterized mostly in nonmammalian hair cells and is observed mainly for hair bundle deflections within the $100 \mathrm{~nm}$ range.

Although the mechanism of fast adaptation remains actively debated, this process has been put forward as a potential mechanism of amplification in nonmammalian hair cells. As transduction channels close, they increase tension in the attached tip links, which pulls the bundle in the negative direction to cause the observed twitch. If appropriately timed, this action could pump energy into the bundle movement (Hudspeth et al. 2000). The gradient in $\mathrm{Ca}^{2+}$ concentration, maintained by ATP-consuming pumps, would provide the requisite energy to power the active movement (Lumpkin and Hudspeth 1998).

The nature of the $\mathrm{Ca}^{2+}$ interaction with the channel and the mechanism of the resulting reclosure is not known (LeMasurier and Gillespie 2005), although a number of models have been proposed (Ricci et al. 2002; Bozovic and Hudspeth 2003; Cheung and Corey 2006). The ions may attach and directly induce a conformational change in the channels. Other models have hypothesized an additional element to be present in the transduction complex, which could be located inside the stereocilia and in series with the tip link. The proposed relaxation element would modulate the overall stiffness by becoming more compliant on the binding of $\mathrm{Ca}^{2+}$ ions. In addition, a voltage-sensitive element has been proposed to contribute to the fast adaptation process (Cheung and Corey 2006).

\section{Additional Control Mechanisms}

A set of in vitro studies has also suggested the need for a calcium feedback element that would operate on timescales that are slow relative to that of the myosin motors' activity cycle (Roongthumskul et al. 2011). The proposed element was motivated by the experimental observation that a significant fraction of spontaneous oscillators display bursts of oscillation, interspersed with quiescent intervals. A model that introduced a variable-stiffness gating spring in series with the tip link, with slow modulation of tension by calcium binding, readily captured the bursting behavior.

Other mechanisms may exist inside a hair cell that control the gain of amplification, finetune the sensitivity, and maintain the robustness of bundle activity. In vivo evidence indicates that exposure to intense sound can temporarily reduce the acuity of hearing (Clark 1991; Melnick 1991). Hence, an internal process adjusts the sensitivity of detection in response to the acoustic environment. It is not known whether some portion of this feedback operates peripherally, at the level of the cochlear hair cells, or if it only occurs at a higher level of processing within the auditory pathways. An experiment performed in vitro explored how deflections of high amplitude, analogous to the effects of intense auditory stimulation, affect the bundle oscillations. Spontaneous oscillations were consistently suppressed by the application of a strong stimulus train (Kao et al. 2013). Innate oscillation robustly returned after cessation of the stimulus, with the duration of the suppression dependent on that of the imposed drive. External calcium con- 
centration impacted the dynamics of suppression and recovery, again implicating calcium in the feedback process.

The molecular identity of the various proposed elements of this feedback process remains unknown. It is possible that different structures are involved with each mechanism and contribute to the response with different dynamics. Alternatively, some of the phenomena could be manifestations of the same process operating in different regimes. In most of the mechanisms studied, calcium strongly affects the dynamics of the feedback and does so at multiple timescales.

\section{HAIR BUNDLE ACTIVITY IN THE MAMMALIAN COCHLEA}

Terrestrial vertebrates studied thus far show similar features in their auditory sensitivity and characteristics, implying that some form of amplification may be common to land vertebrates (Manley 2000). Although the presence of an active amplifier in hearing organs seems ubiquitous, its precise nature and its variation across species have not been established.

Hair bundle dynamics have been most extensively explored in hair cells of the amphibian sacculus, an organ that is sufficiently robust to allow prolonged mechanical probing of the active response. The applicability of these findings to the mechanics of the cochlea remains an open question. A large body of experimental evidence favors somatic electromotility of the mammalian outer hair cells as the primary motor of amplification in the cochlea (see Ashmore 2018). The potential contribution of active bundle motility to cochlear amplification, or whether an interaction between the two mechanisms jointly shapes the cochlear response, has not been established, and the topic remains an area of current research.

Experimental evidence does indicate several phenomena in the cochlear hair bundle response that are analogous to those observed in other species. The process of fast adaptation was shown in the rat cochlea (Kennedy et al. 2003). Outer hair cells were patch-clamped, and the transduction current was measured while the bundles were deflected with stimulus probes.
The adaptation time constant, with corrections for temperature and the endocochlear potential, was estimated to be $\sim 50 \mu \mathrm{sec}$. The process was found to resemble fast adaptation observed in nonmammalian systems. Another study performed on isolated cochleae of neonatal rats showed a nonlinear force-displacement relationship (Kennedy et al. 2005). The nonlinearity was shown to develop with a time course that was similar to fast adaptation. Finally, a power-law response at the level of the hair bundle was shown in vitro on preparations of the gerbil cochlea (Chan and Hudspeth 2005). The mechanical response showed a resonance, indicative of frequency selectivity in the system. Hence, several signatures of an active nonlinear response have been established in the mammalian hair bundles.

\section{THEORETICAL MODELS}

\section{Biophysical Models of Active Bundle Motility}

Spontaneous hair bundle oscillations can be successfully described by models that combine the transduction and adaptation processes (Hudspeth 2008). The opening and closing of mechanically sensitive ion channels generates the negative stiffness region. In parallel, an array of molecular motors is assumed to continuously shift the operating point of the transduction complex. When the channels are closed, the adaptation motors climb along the actin filaments of the hair bundles, increasing the tension in the tip links. At a critical tension, the channels open, allowing the influx of $\mathrm{K}^{+}$, the main transduction ion, and $\mathrm{Ca}^{2+}$, which carries a small fraction of the current. The presence of calcium and the imposed tension cause the myosin motors to slip down the actin filaments. As the tension relaxes, the ion channels close, and the process repeats itself.

This interplay between the negative gating stiffness and adaptation can be readily reproduced by the following equations (Martin et al. 2003). The hair bundle is assumed to operate in the overdamped regime in which inertia plays no role, and hence the mass term is negligible. The net force experienced by the bundle includes the 


\section{Bozovic}

viscous drag term, the extension of the gating springs, and deflection of the stereocilia:

$\xi \frac{d X}{d t}=-N \gamma k_{g s}\left(\gamma X-x_{a}-p_{o} d\right)-K_{s p}\left(X-X_{s p}\right)$

where $X$ is the position of the bundle, $x_{a}$ is the position of the adaptation motor along the length of the stereocilium, $\xi$ is the viscous drag of the bundle, $N$ is the number of channels, $\gamma$ is a geometric projection factor, $d$ is the effective swing distance of the channel gate, $k_{g s}$ is the stiffness of the gating spring, $K_{s p}$, and $X_{s p}$ is an offset term. The term describing the extension of the gating springs summarizes a number of findings obtained from in vitro measurements of hair bundle mechanics. Deflection $(X)$ of the stereocilia increases tension on the gating spring, while slippage $\left(x_{a}\right)$ of the adaptation motors and the effective extension $\left(p_{o} d\right)$ caused by opening of the channels reduces it. The opening probability of the channels is simply described by the Boltzmann distribution:

$p_{o}(X)=\frac{1}{1+e\left[\Delta E^{0}-k_{g s} d\left(\gamma X-x_{a}-d / 2\right)\right] /(k T)}$,

where $\Delta E^{0}$ describes an innate energy difference between the closed and open states, $k$ is the Boltzmann constant, and $T$ is the temperature. The dynamics of the myosin motors are described by:

$$
\frac{d x_{a}}{d t}=-C+S k_{g s}\left(\gamma X-x_{a}-p_{o} d\right)
$$

where $C$ and $S$ are the climbing and slipping rates of the myosin motors, respectively. They are assumed to be linearly dependent on the binding of calcium ions to a local binding site, with association and dissociation of calcium governed by the standard mass-action rate equations.

Several variants of the above model have been explored in the literature (Nadrowski et al. 2004; Han and Neiman 2010). Specific terms contributing to the total force offset are sometimes explicitly included (Martin et al. 2000). Additional terms often include an extension spring, which limits the full range of the adaptation. Finally, modulation of the gating spring, showing either fast (Bozovic and Hudspeth 2003) or slow (Roongthumskul et al. 2011) dynamics, has been modeled by equations that express this stiffness as a simple function of calcium binding. This model summarizes a large body of experimental findings obtained from actively oscillating hair bundles, and robustly reproduces the observed dynamics.

\section{Nonlinear Dynamics and Bifurcation Theory}

Biological processes are frequently highly nonlinear, as well as active, requiring a continuous supply of energy to maintain function. The branch of mathematics known as nonlinear dynamics has developed a general approach for the study of such systems. The beauty of these mathematical theories is that they not only provide techniques for solving specific systems of equations, but more importantly, they uncover universal features and phenomena that are common to whole classes of nonlinear systems. Hence, they provide powerful simplification tools: elaborate nonlinear models can be mapped onto much simpler, so-called normal form equations (Strogatz 1994). This allows for modeling with much sparser a priori assumptions, far fewer free parameters, and ultimately similar predictions, independent of the details of the specific system considered.

The cubic nonlinearity showed by the inner ear maps onto a well-known equation, known in physics as the Stuart-Landau equation, which has been extensively studied in a number of physical systems (Pikovsky et al. 2001). The solution to this equation shows a bifurcation, a qualitative change in behavior when an internal control parameter is varied. This bifurcation, known as the Hopf bifurcation (also called Andronov-Hopf), corresponds to a critical point where the system drastically changes behavior. For control parameters below this critical value, the system is quiescent, that is, does not show motion in the absence of stimulus; above the critical value, it oscillates spontaneously. In the vicinity of the bifurcation, the system is extremely sensitive, showing both amplification and 


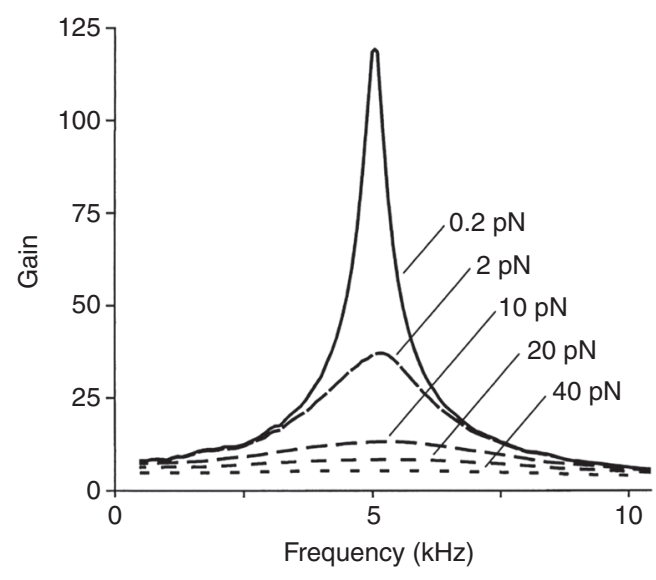

Figure 4. Theoretical predictions based on the Hopf bifurcation model. For a system near the critical point, the response was shown to be highly frequency selective and to show a high gain of amplification with characteristic one-third power-law nonlinearity. As the stimulus amplitude increased, the response broadened, and the gain decreased. (From Choe et al. 1998; reprinted, with permission, from the National Academy of Sciences (C) 1998.)

high-frequency selectivity (Fig. 4). Away from the bifurcation, the response sensitivity decreases, and the frequency tuning broadens. This simple theoretical framework was shown to capture many complex phenomena shown by the inner ear (Choe et al. 1998; Eguiluz et al. 2000; Duke and Julicher 2003; Kern and Stoop 2003; Magnasco 2003).

The Hopf bifurcation was shown to be consistent with the main features observed in measurements of hair bundle motility (Martin and Hudspeth 2001; Martin et al. 2001), even including the dynamics of small fluctuations in the bundle position (Sheth et al. 2018). A theoretical study applied bifurcation analysis to a simplified version of the model of bundle dynamics, discussed above (Nadrowski et al. 2004). The model included mechanoelectrical transduction, channel gating, adaptation, and calcium feedback. Varying certain key parameters in the model led to different solutions to the sets of equations, leading to different regimes of dynamical behavior. The state diagram predicted lines of Hopf bifurcation, separating the spontaneously oscillating and quiescent regimes. This state diagram
Hair Bundles

was subsequently verified experimentally (Salvi et al. 2015). A dynamical clamp was constructed, which allowed one to monitor hair bundle motility in real time and to apply mechanical feedback so as to mimic a consistent stiffness load, as well as an imposed offset force. These two parameters were varied over a broad range and allowed the reconstruction of the state diagram predicted theoretically. The transitions from oscillation to quiescence showed all the features of the Hopf bifurcation, lending strong experimental support to the theory.

Other types of bifurcations have also been identified in the response of actively oscillating hair bundles. An experimental study measured the bundle response to stimuli applied across the full physiological range of amplitudes and frequencies (Fredrickson-Hemsing et al. 2012). Performed in vitro on hair bundles from the bullfrog sacculus, the experiments showed that the phase-locked component of the oscillation displays a triangular shape, with peaked response occurring at the characteristic frequency of the cell, and with tuning that broadened with increasing stimulus amplitude. This shape of response is known in nonlinear dynamics literature as an Arnold tongue (Pikovsky et al. 2001), and can be readily reproduced by a simple theoretical model. The model based on the normal form equation predicted the occurrence of the Hopf bifurcation, but also that of the "saddle node on invariant circle" (SNIC) bifurcation, as well as others. The SNIC was shown to be the bifurcation that dominates the response of the hair bundle to weak stimuli. Individual hair bundles that show spontaneous oscillation seem barely affected by the imposition of a stimulus of amplitude in the range of a nanometer or smaller. However, the phase of the oscillation is affected even by such minute signals: although full entrainment is not reached, the bundle shows periods of phase-locking interspersed with phase slips of $2 \pi$ (Roongthumskul et al. 2013). This behavior is fully reproduced by a phase dynamics equation that describes the SNIC bifurcation. The occurrence of other bifurcations in the response of a hair cell has been reproduced in more complex models (Maoiléidigh et al. 2012). 


\section{Bozovic}

An important insight provided by the comparison between the mathematical models and the biological cells is that changes in the control parameters can drastically affect the dynamics of the cell's behavior. For example, in contrast to the Hopf bifurcation, the SNIC bifurcation is not frequency selective. However, it is highly sensitive to small stimuli, even in the presence of noise. Future work in this direction may allow us to identify more clearly how changes in the biophysics of the hair cell may bias its behavior toward vestibular or auditory detection characteristics, what determines its sharpness of frequency tuning or gain of amplification, and what are the key parameters that shift when these properties are degraded.

\section{Self-Tuning and Gain Control}

One limitation of the Hopf bifurcation model is that the control parameter, which determines the level of amplification, frequency selectivity, etc., is a constant. The auditory system is, on the contrary, likely to modulate this parameter in response to external sounds. An early theoretical study had proposed that a system poised near the Hopf bifurcation would self-tune to its vicinity in a dynamical fashion (Camalet et al. 2000). A later model built on this notion to explain the experimental observation of suppression and recovery of oscillation. It was proposed that the control parameter shifts away from the bifurcation and into the quiescent regime, upon prolonged exposure to strong stimuli (Shlomovitz et al. 2013). On cessation of the drive, a feedback process would reverse the effect, and tune the hair cell back into the oscillatory state. Inclusion of a dynamic control parameter into the Hopf model was shown to lead to a regime of chaotic oscillations, consistent with experimental measurements of the bundle (Faber and Bozovic 2017).

The presence of an internal feedback process in the hair cell is both plausible and biologically very attractive. It obviates the need for unrealistically precise control during development to poise the cells near criticality. Further, it could serve to protect the delicate hair cell machinery from damage during exposure to loud sounds by detuning the cells into a less sensitive regime. The biophysical mechanisms of this process remain, however, to be discovered, and the proposed hypotheses await definitive experimental proof.

\section{ARRAYS OF COUPLED HAIR BUNDLES}

The inner ear organs of different species show a variety of coupling structures (Manley 2000). Although hair bundles of some end organs are free-standing, others are coupled, either in small groups by sallets, as in the lizard papilla, or in large numbers, by membranes that span most or all of the sensory epithelium. There are variations in the thickness and shape of the overlying membranes, and hence in the strength of the imposed coupling. In the bullfrog sacculus, the otolithic membrane, a homogeneous matrix of cross-linked filaments, strongly couples the hair bundles without impeding deflections in the lateral direction (Kachar et al. 1990). The mammalian tectorial membrane was shown to be more heterogeneous in its properties, with transverse thickness decreasing and the width increasing from the base to the apex of the cochlea, coding for high and low acoustic frequencies, respectively (Freeman et al. 2003). The varying forms and extents of coupling in different end organs raise the question of how exactly this coupling affects the dynamics of hair bundles.

A theoretical study explored systems of many coupled nonlinear oscillators, each poised in the vicinity of a Hopf bifurcation (Risler et al. 2004). With the introduction of noise into the system, the critical point of each individual oscillator was smeared, thus obscuring the bifurcation. However, a system of many oscillators arranged in a lattice, displaying nearest-neighbor interaction, was shown to undergo synchronization over large distances and showed clear signatures of the bifurcation. Comparison to the measurements of the mammalian cochlea, which indicated an approximate one-third power-law dependence over orders of magnitude of stimulus amplitude, indicated that many coupled oscillators synchronize to bring the system close to the critical point. Another study explored a more detailed biophysical model of hair bundle 
mechanics and introduced elastic springs as coupling elements between nearest neighbors (Dierkes et al. 2008). The model indicated that the sensitivity of the system, as well as the range and extent of nonlinearity, was enhanced by the coupling.

An experimental study also tested the effects of "coupling" a hair bundle to its cyber clone (Barral et al. 2010). Real-time monitoring of a biological hair bundle was used to virtually couple it (by use of a feedback system) to a numerical simulation that mimicked its dynamics. A control parameter setting the strength of the coupling was then continuously adjusted to test the effects on the overall response. With progressively higher coupling, the real cell and the virtual one synchronized their spontaneous oscillations, and the quality factor of the oscillations was observed to increase. The sensitivity of the coupled system increased, and the dynamic range over which the compressive nonlinearity was observed broadened. Hence, coupling between hair bundles may serve an important role in sharpening their overall mechanical response.

\section{CONCLUDING REMARKS}

This review summarizes some of the progress made in understanding the cellular mechanisms underlying hair bundle activity, as well as some of the theoretical models that have been designed to explain the experimental observations. Mechanoelectrical transduction has been extensively studied and characterized, as have the different adaptation processes operating within a hair bundle. Theoretical models based on the biophysical processes hypothesized to be at work inside the hair cell have reproduced many phenomena, including spontaneous oscillations, rapid active twitch phenomena, effects of calcium feedback, and response to electrical stimuli. Many questions still remain open, however, providing avenues for future research.

Nonlinear dynamics theory has provided a powerful mathematical language in which to describe and explore the detection characteristics of the inner ear's mechanosensing units. Significant experimental support for the Hopf bifurca- tion theory of auditory detection has been gathered, showing consistency with the theoretical predictions. However, a definitive experimental proof of this theory still awaits an unambiguous identification of the biological control parameter proposed to continuously tune the dynamic state of the bundle and a demonstration of an enhanced sensitivity in the vicinity of a critical point.

Second, the mechanisms of amplification are still not fully resolved, especially in the cochlea where the respective contributions of outer hair cell somatic motility and active bundle movements to the amplification process remain unclear. The dynamics that determine the frequency selectivity of an individual cell and the overall frequency range of an end organ are likewise not clear. Comparative studies are likely to yield further insight into the features that are conserved across the various species, and the mechanisms that are unique to the mammalian cochlea.

Third, the pertinence of the notion of selftuning of the amplifier, and its potential role in maintaining hair cell integrity over a broad dynamic range of incoming sound, is not known. Establishing the biological mechanisms of this dynamic gain control, and in particular, clarifying how such a self-tuning mechanism might be connected to the control of amplification exerted by the efferent system, are some of the main challenges of future research.

Fourth, the role of the coupling between hair bundles in determining their contribution to the response of the full end organ needs to be more extensively explored. Coupling may be the most plausible mechanism for defeating noise in the system, and several theoretical studies have shown its potential benefits. The theoretical models await explicit experimental verification and may provide a connection between our current understanding of hair bundle dynamics and the observed in vivo phenomena.

\section{ACKNOWLEDGMENTS}

The author thanks Justin Faber, Janaki Sheth, and Jessica Lin for helpful comments on the manuscript. 
D. Bozovic

\section{REFERENCES}

* Reference is also in this collection.

* Ashmore J. 2018. Outer hair cells and electromotility. Cold Spring Harb Perspect Med doi: 10.1101/cshperspect. a033522.

Barral J, Martin P. 2012. Phantom tones and suppressive masking by active nonlinear oscillation of the hair-cell bundle. Proc Natl Acad Sci 109: E1344-E1351.

Barral J, Dierkes K, Lindner B, Julicher F, Martin P. 2010. Coupling a sensory hair-cell bundle to cyber clones enhances nonlinear amplification. Proc Natl Acad Sci 107: 10765-10765.

Benser ME, Marquis RE, Hudspeth AJ. 1996. Rapid, active hair bundle movements in hair cells from the bullfrog's sacculus. J Neurosci 16: 5629-5643.

Bozovic D, Hudspeth AJ. 2003. Hair-bundle movements elicited by transepithelial electrical stimulation of hair cells in the sacculus of the bullfrog. Proc Natl Acad Sci 100: $958-963$.

Camalet S, Duke T, Julicher F, Prost J. 2000. Auditory sensitivity provided by self-tuned critical oscillations of hair cells. Proc Natl Acad Sci 97: 3183-3188.

Chan DK, Hudspeth AJ. 2005. Ca ${ }^{2+}$ current-driven nonlinear amplification by the mammalian cochlea in vitro. Nat Neurosci 8: 149-155.

Cheung EL, Corey DP. 2006. $\mathrm{Ca}^{2+}$ changes the force sensitivity of the hair-cell transduction channel. Biophys J 90: 124-139.

Choe Y, Magnasco MO, Hudspeth AJ. 1998. A model for amplification of hair-bundle motion by cyclical binding of $\mathrm{Ca}^{2+}$ to mechanoelectrical-transduction channels. Proc Natl Acad Sci 95: 15321-15326.

Clark WW. 1991. Recent studies of temporary threshold shift (TTS) and permanent threshold shift (PTS) in animals. J Acoust Soc Am 90: 155-163.

Crawford AC, Fettiplace R. 1985. The mechanical properties of ciliary bundles of turtle cochlear hair cells. J Physiol 364: 359-379.

Dallos P. 1992. The active cochlea. J Neurosci 12: 4575-4585.

Dierkes K, Lindner B, Julicher F. 2008. Enhancement of sensitivity gain and frequency tuning by coupling of active hair bundles. Proc Natl Acad Sci 105: 18669-18674.

Dinis L, Martin P, Barral J, Prost J, Joanny JF. 2012. Fluctuation-response theorem for the active noisy oscillator of the hair-cell bundle. Phys Rev Lett 109: 160602-160605.

Duke T, Julicher F. 2003. Active traveling wave in the cochlea. Phys Rev Lett 90: 158101.

Eatock RA. 2000. Adaptation in hair cells. Annu Rev Neurosci 23: 285-314.

Eguiluz VM, Ospeck M, Choe Y, Hudspeth AJ, Magnasco MO. 2000. Essential nonlinearities in hearing. Phys Rev Lett 84: 5232-5235.

Faber J, Bozovic D. 2017. Chaotic dynamics of hair cells. Sci Rep 8: 3366.

Fettiplace R, Hackney CM. 2006. The sensory and motor roles of auditory hair cells. Nat Rev 7: 19-29.

Fettiplace R, Ricci AJ. 2003. Adaptation in auditory hair cells. Curr Opin Neurobiol 13: 446-451.
Fredrickson-Hemsing L, Ji S, Bruinsma R, Bozovic D. 2012. Mode-locking dynamics of hair cells of the inner ear. Phys Rev E 86: 021915.

Freeman DM, Masaki K, McAllister AR, Wei JL, Weiss TF. 2003. Static material properties of the tectorial membrane: A summary. Hear Res 180: 11-27.

Furness DN, Mahendrasingam S, Ohashi M, Fettiplace R, Hackney CM. 2008. The dimensions and composition of stereociliary rootlets in mammalian cochlear hair cells: Comparison between high- and low-frequency cells and evidence for a connection to the lateral membrane. J Neurosci 28: 6342-6353.

Gillespie PG, Corey DP. 1997. Myosin and adaptation by hair cells. Neuron 19: 955-958.

Gillespie PG, Cyr JL. 2004. Myosin-1c, the hair cell's adaptation motor. Annu Rev Physiol 66: 521-545.

Gillespie PG, Muller U. 2009. Mechanotransduction by hair cells: Models, molecules, and mechanisms. Cell 139: 3344.

Gillespie PG, Dumont RA, Kachar B. 2005. Have we found the tip link, transduction channel, and gating spring of the hair cell? Curr Opin Neurobiol 15: 389-396.

Gold T. 1948. Hearing. II: The physical basis of the action of the cochlea. Proc R Soc Lond B 135: 492-498.

Han LJ, Neiman AB. 2010. Spontaneous oscillations, signal amplification, and synchronization in a model of active hair bundle mechanics. Phys Rev E 81: 041913.

Holt JR, Corey DP. 2000. Two mechanisms for transducer adaptation in vertebrate hair cells. Proc Natl Acad Sci 97: 11730-11735.

Holt JR, Gillespie SKH, Provance DW, Shah K, Shokat KM, Corey DP, Mercer JA, Gillespie PG. 2002. A chemicalgenetic strategy implicates myosin-1c in adaptation by hair cells. Cell 108: 371-381.

Howard J, Hudspeth AJ. 1988. Compliance of the hair bundle associated with gating of mechanoelectrical transduction channels in the bullfrog's saccular hair cell. Neuron 1: 189-199.

Hudspeth AJ. 2000. Hearing. In Principles of neural science (ed. Kandel ER, Schwartz JH, Jessell TM), pp. 590-624. McGraw-Hill, New York.

Hudspeth AJ. 2008. Making an effort to listen: Mechanical amplification in the ear. Neuron 59: 530-545.

Hudspeth AJ. 2014. Integrating the active process of hair cells with cochlear function. Nat Rev Neurosci 15: 600614.

Hudspeth AJ, Gillespie PG. 1994. Pulling springs to tune transduction: Adaptation by hair cells. Neuron 12: 1-9.

Hudspeth AJ, Choe Y, Mehta AD, Martin P. 2000. Putting ion channels to work: Mechanoelectrical transduction, adaptation, and amplification by hair cells. Proc Natl Acad Sci 97: 11765-11772.

Jaramillo F, Markin VS, Hudspeth AJ. 1993. Auditory illusions and the single hair cell. Nature 364: 527-529.

Kachar B, Parakkal M, Fex J. 1990. Structural basis for mechanical transduction in the frog vestibular sensory apparatus. I: The otolithic membrane. Hear Res 45: 179-190.

Kao A, Meenderink SW, Bozovic D. 2013. Mechanical overstimulation of hair bundles: Suppression and recovery of active motility. PLoS ONE 8: e58143. 
Kennedy HJ, Evans MG, Crawford AC, Fettiplace R. 2003 Fast adaptation of mechanoelectrical transducer channels in mammalian cochlear hair cells. Nat Neurosci 6: 832836.

Kennedy HJ, Crawford AC, Fettiplace R. 2005. Force generation by mammalian hair bundles supports a role in cochlear amplification. Nature 433: 880-883.

Kern A, Stoop R. 2003. Essential role of couplings between hearing nonlinearities. Phys Rev Lett 91: 128101.

Kitajiri S, Sakamoto T, Belyantseva IA, Goodyear RJ, Stepanyan R, Fujiwara I, Bird JE, Riazuddin S, Ahmed ZM, Hinshaw JE, et al. 2010. Actin-bundling protein TRIOBP forms resilient rootlets of hair cell stereocilia essential for hearing. Cell 141: 786-798.

Kozlov AS, Risler T, Hudspeth AJ. 2007. Coherent motion of stereocilia assures the concerted gating of hair-cell transduction channels. Nat Neurosci 10: 87-92.

Le Goff L, Bozovic D, Hudspeth AJ. 2005. Adaptive shift in the domain of negative stiffness during spontaneous oscillation by hair bundles from the internal ear. Proc Natl Acad Sci 102: 16996-17001.

LeMasurier M, Gillespie PG. 2005. Hair-cell mechanotransduction and cochlear amplification. Neuron 48: 403-415.

Lumpkin EA, Hudspeth AJ. 1998. Regulation of free $\mathrm{Ca}^{2+}$ concentration in hair-cell stereocilia. J Neurosci 18: 63006318.

Magnasco MO. 2003. A wave traveling over a Hopf instability shapes the cochlear tuning curve. Phys Rev Lett 90: 058101.

Manley GA. 2000. Cochlear mechanisms from a phylogenetic viewpoint. Proc Natl Acad Sci 97: 11736-11743.

Manley GA, Koppl C. 1998. Phylogenetic development of the cochlea and its innervation. Curr Opin Neurobiol 8: 468-474.

Maoiléidigh DO, Nicola E, Hudspeth AJ. 2012. The diverse effects of mechanical loading on active hair bundles. Proc Natl Acad Sci 109: 1943-1948.

Markin VS, Hudspeth AJ. 1995. Gating-spring models of mechanoelectrical transduction by hair cells of the internal ear. Annu Rev Biophys Biomol Struct 24: 59-83.

Martin P, Hudspeth AJ. 1999. Active hair-bundle movements can amplify a hair cell's response to oscillatory mechanical stimuli. Proc Natl Acad Sci 96: 14306-14311.

Martin P, Hudspeth AJ. 2001. Compressive nonlinearity in the hair bundle's active response to mechanical stimulation. Proc Natl Acad Sci 98: 14386-14391.

Martin P, Mehta AD, Hudspeth AJ. 2000. Negative hairbundle stiffness betrays a mechanism for mechanical amplification by the hair cell. Proc Natl Acad Sci 97: 1202612031.

Martin P, Hudspeth AJ, Julicher F. 2001. Comparison of a hair bundle's spontaneous oscillations with its response to mechanical stimulation reveals the underlying active process. Proc Natl Acad Sci 98: 14380-14385.

Martin P, Bozovic D, Choe Y, Hudspeth AJ. 2003. Spontaneous oscillation by hair bundles of the bullfrog's sacculus. J Neurosci 23: 4533-4548.

Meenderink SWF, Quinones PM, Bozovic D. 2015. Voltagemediated control of spontaneous bundle oscillations in saccular hair cells. J Neurosci 35: 14457-14466.
Melnick W. 1991. Human temporary threshold shift (TTS) and damage risk. J Acoust Soc Am 90: 147-154.

Nadrowski B, Martin P, Julicher F. 2004. Active hair-bundle motility harnesses noise to operate near an optimum of mechanosensitivity. Proc Natl Acad Sci 101: 1219512200.

* Petit C. 2018. Hair-bundle links: Genetics as the gateway to function. Cold Spring Harb Perspect Med doi: 10.1101/ cshperspect.a033142.

Pikovsky A, Rosenblum M, Kurths J. 2001. Synchronization: A universal concept in nonlinear sciences. Cambridge University Press, Cambridge, UK.

Reichenbach T, Hudspeth AJ. 2014. The physics of hearing: Fluid mechanics and the active process of the inner ear. Rep Prog Phys 77: 076601.

Ricci AJ, Crawford AC, Fettiplace R. 2002. Mechanisms of active hair bundle motion in auditory hair cells. J Neurosci 22: $44-52$.

Risler T, Prost J, Julicher F. 2004. Universal critical behavior of noisy coupled oscillators. Phys Rev Lett 93: 175702.

Robles L, Ruggero MA. 2001. Mechanics of the mammalian cochlea. Physiol Rev 81: 1305-1352.

Roongthumskul Y, Fredrickson-Hemsing L, Kao A, Bozovic D. 2011. Multiple-timescale dynamics underlying spontaneous oscillations of saccular hair bundles. Biophys J 101: 603-610.

Roongthumskul Y, Shlomovitz R, Bruinsma R, Bozovic D. 2013. Phase slips in oscillatory hair bundles. Phys Rev Lett 110: 148103 .

Russell IJ, Kossl M, Richardson GP. 1992. Nonlinear mechanical responses of mouse cochlear hair bundles. Proc Biol Sci 250: 217-227.

Salvi J, O Maoiléidigh D, Fabella BA, Tobin M, Hudspeth AJ. 2015. Control of a hair bundle's mechanosensory function by its mechanical load. Proc Natl Acad Sci 112: E1000-E1009.

Sheth J, Meenderink SWF, Quiñones PM, Bozovic D, Levine AJ. 2018. Nonequilibrium limit-cycle oscillators: Fluctuations in hair bundle dynamics. Phys Rev E 97: 062411.

Shlomovitz R, Fredrickson-Hemsing L, Kao A, Meenderink SW, Bruinsma R, Bozovic D. 2013. Low frequency entrainment of oscillatory bursts in hair cells. Biophys 104: 1661-1669.

Sotomayor M, Weihofen WA, Gaudet R, Corey DP. 2010. Structural determinants of cadherin-23 function in hearing and deafness. Neuron 66: 85-100.

Strogatz SH. 1994. Nonlinear dynamics and chaos. Westview, Cambridge, MA.

van der Heijden M. 2014. Frequency selectivity without resonance in a fluid waveguide. Proc Natl Acad Sci 111: 14548-14552.

van Netten SM, Dinklo T, Marcotti W, Kros CJ. 2003. Channel gating forces govern accuracy of mechano-electrical transduction in hair cells. Proc Natl Acad Sci 100: 15510 15515.

Vollrath MA, Kwan KY, Corey DP. 2007. The micromachinery of mechanotransduction in hair cells. Annu Rev Neurosci 30: 339-365. 


\section{$\&_{\mathrm{CSH}}^{\infty} \&$ Cold Spring Harbor

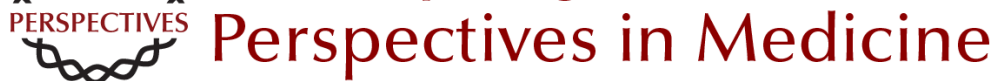

\section{Active Biomechanics of Sensory Hair Bundles}

Dolores Bozovic

Cold Spring Harb Perspect Med 2019; doi: 10.1101/cshperspect.a035014 originally published online October 15, 2018

\section{Subject Collection Function and Dysfunction of the Cochlea}

Hidden Hearing Loss: A Disorder with Multiple Etiologies and Mechanisms

David C. Kohrman, Guoqiang Wan, Luis Cassinotti, et al.

Hair Cell Afferent Synapses: Function and Dysfunction

Stuart L. Johnson, Saaid Safieddine, Mirna Mustapha, et al.

Active Biomechanics of Sensory Hair Bundles Dolores Bozovic

The Tectorial Membrane: Mechanical Properties and Functions

Jonathan B. Sellon, Roozbeh Ghaffari and Dennis M. Freeman

The Epidemiology of Deafness Abraham M. Sheffield and Richard J.H. Smith

Toward the Optical Cochlear Implant Tobias Dombrowski, Vladan Rankovic and Tobias Moser

Outer Hair Cells and Electromotility Jonathan Ashmore

Interactions between Macrophages and the Sensory Cells of the Inner Ear Mark E. Warchol
Development and Patterning of the Cochlea: From Convergent Extension to Planar Polarity Mireille Montcouquiol and Matthew W. Kelley

Hair-Bundle Links: Genetics as the Gateway to Function

Guy P. Richardson and Christine Petit

Aminoglycoside- and Cisplatin-Induced

Ototoxicity: Mechanisms and Otoprotective

Strategies

Corné J. Kros and Peter S. Steyger

Function and Dysfunction of TMC Channels in Inner Ear Hair Cells

David P. Corey, Nurunisa Akyuz and Jeffrey R. Holt

Cochlear Gene Therapy

Lawrence Lustig and Omar Akil

Age-Related Hearing Loss

Michael R. Bowl and Sally J. Dawson

Inner Ear Connexin Channels: Roles in

Development and Maintenance of Cochlear

Function

Fabio Mammano

A Functional Perspective on the Evolution of the Cochlea Christine Köppl and Geoffrey A. Manley

For additional articles in this collection, see http://perspectivesinmedicine.cshlp.org/cgi/collection/ 